\title{
Un cuestionamiento de los supuestos que vertebran la sociedad moderna en búsqueda de nuevos modelos de gobernanza: el gobierno, la sociedad civil y la empresa nadando en un mar de complejidad
}

\author{
Sergio GARCÍA MAGARIÑO \\ Universidad Camilo José Cela \\ tresycinco@gmail.com
}

Recibido: 14/09/2015

Aceptado: 19/11/2015

\begin{abstract}
Resumen
Con este artículo se pretenden tres objetivos. El primero consiste en hacer una aproximación introductoria al concepto de gobernanza, con el fin de establecer un lenguaje común desde el que abordar sus múltiples facetas en colaboración con otros autores. El segundo es plantear algunas preguntas marco relativas a la gobernanza que puedan guiar la reflexión de diferentes actores, de forma que no sean aportaciones fragmentadas sino diferentes componentes de un acervo de conocimiento coherente. Por último, se aspira a iniciar una exploración de las preguntas planteadas atendiendo a las implicaciones prácticas para la gobernanza de ciertos principios. En este sentido, como en otras ocasiones, la ciencia y la religión son consideradas dos fuentes de las que adquirir percepciones para encontrar modelos más efectivos de organización y desarrollo social. El artículo se fundamenta en la ponencia inaugural que la oficina de asuntos públicos de la comunidad bahá'í de España realizó en la jornada celebrada el día 22 de mayo de 2014 en el Centro de Estudios Políticos y Constitucionales titulada «El imperativo de la colaboración: el gobierno, la sociedad civil y la empresa navegando en un mar de complejidad». Consta de cuatro partes: 1. ¿A qué nos referimos por gobernanza? 2. Propósito de la jornada. 3. Sectores y niveles de gobernanza y preguntas marco. 4. Cuestionando algunos principios subyacentes.
\end{abstract}

Palabras clave: gobernanza, organización social, principios sociales, participación, colaboración.

Questioning the assumptions underpinning modern society in a search for new patterns of governance: government, civil society and companies sailing in a sea of complexity

\begin{abstract}
This paper has three main aims. First, to make an introduction about the concept of governance in order to generate a common language from which to approach, in collaboration with other authors, its manifold facets. Second, to propose a framework with certain questions that, on the one hand, enable different kind of actors to reflect on this important topic - governance - and, on the other, allow for integrating the knowledge generated in this field into a coherent body. Finally, the paper attempts to respond to some of the questions posed at the beginning by looking at the practical implications on the governance mechanisms of certain principles. In this last respect, the underlying assumption is that there must be a dialogue bet-
\end{abstract}


ween science and religion to find more effective models of social organization and development. The paper is grounded on the opening remark that the Office or Public Affairs of the Baha'i community of Spain presented in the conference «The paramount need for cooperation: the government, the civil society and the private sector navigating on a sea of complexity» held in the Centro de Estudios Políticos y Constitucionales of Madrid the 22th of May of 2014. The article is divided into four sections: 1 . What is meant by governance? 2 . The purpose of the conference. 3. Sectors and levels of governance. 4 . Calling into question some underlying tenets.

Key words: governance, social organization, social principles, participation, collaboration.

\section{1. ¿A qué nos referimos por gobernanza?}

La gobernanza es un concepto relativamente nuevo sobre cuyo significado hay cierto debate. Por gobernanza nos referimos, de un lado, a la constatación de una crisis, de ciertas transformaciones sociales y de un agotamiento de los principios organizativos de la vida social en general y del gobierno en particular. En las últimas décadas se han producido transformaciones sociales de tal calado que parecen indicar la emergencia de una nueva realidad social. Algunas de las manifestaciones más claras de estas transformaciones son la pérdida de capacidad de la política para responder a la creciente complejidad social. Las elecciones democráticas constituían anteriormente el elemento definitivo de la legitimidad de un gobierno, pero hoy día parece no ser suficiente. La sociedad civil, mucho más inteligente y organizada que antaño, reclama mayor transparencia y mayor participación en la toma de decisiones, y exige una ética más elevada por parte de los gobernantes. Los partidos políticos, la religión y la clase social, aspectos que eran claves en la formación de las identidades colectivas, estructuraban y fomentaban la cohesión social; algo que actualmente ya no ocurre. Las sociedades y las relaciones entre los individuos, entre las instituciones y entre individuos e instituciones - por señalar algunas-, en el pasado ocurrían principalmente dentro del confín del Estado-nación, y las decisiones más importantes en materia económica o política también se daban allí. No obstante, en la actualidad los fenómenos sociales y las relaciones se han globalizado y los espacios para la toma de decisiones se han multiplicado, sobrepasando en muchos casos el nivel nacional. Muchos países -especialmente en la Unión Europea- reconocen que un porcentaje muy alto de las decisiones más importante que afectan a sus territorios se toman en los ámbitos supranacionales, para los cuales, mayormente, no hay instituciones legítimamente elegidas. Por último, los principios que nutrían las prácticas de gestión y administración pública y privada de jerarquía, centralización y competencia, y el clima de seguridades y certezas en el que se tomaban las decisiones, parecen haber agotado su capacidad de dirigir una sociedad más compleja, organizada, interdependiente e inteligente cuya gestión parece requerir grados de colaboración, descentralización, aprendizaje y tolerancia a la ambigüedad inusitados hasta la fecha.

De otro lado, gobernanza no se refiere al gobierno formal exclusivamente, sino a una forma de dar dirección a la sociedad en la que el gobierno formal, la sociedad civil y el tejido empresarial se entrelazan para gestionar los asuntos públicos. En cierto sen- 
tido, la gobernanza se refiere al hecho de gobernar en un modo distinto, un modo en el que el gobierno se convierte en agente de configuración de espacios sociales donde el resto de actores interactúan y aspiran a regir el curso de la sociedad.

La gobernabilidad y la gobernanza, tal como menciona Luis Fernández Aguilar (2007) no son lo mismo. La gobernabilidad se refiere a las capacidades del gobierno formal para gobernar, capacidades que pasan por mejorar el funcionamiento de la administración pública y la formación del funcionariado, y por introducir el conocimiento científico y técnico al proceso de toma de decisiones, entre otras cosas. La gobernanza, en cambio, aunque incluye el gobierno formal y reconoce la necesidad de estas capacidades, se ocupa también de la forma en que las organizaciones de la sociedad civil y las empresas se organizan internamente, así como, sobre todo, de las maneras en que contribuyen a la gestión de lo que algunos llaman la «cosa pública». De este modo, el concepto de gobernanza es más amplio que el de gobernabilidad y, a pesar de que algunos consideran que es un anglicismo, alude a otra realidad mayor que la indicada por el concepto de gobernabilidad.

Judith E. Innes y David E. Booher en su Planning with complexity muestran algunos casos paradigmáticos, como el de Sacramento, donde la administración pública tuvo que involucrar a los actores en el proceso de toma de decisiones acerca de cómo canalizar las aguas del río de forma satisfactoria para la población. Así lograron solucionar un problema insalvable que estaba durando años y que ningún gobierno local podía resolver. En esta obra, intentando desarrollar una teoría de la racionalidad colaborativa, los autores esbozan lo que se quiere decir por gobernar en modo de gobernanza.

El reciente proyecto del gobierno irlandés para reformar su constitución, «Convention on the Constitution», también construye sobre esta tradición, ya que, durante un año, representantes de la sociedad civil y del mundo empresarial elegidos al azar, debatieron con representantes políticos, tras sesiones educativas conducidas por expertos y académicos, acerca de los temas más relevantes para la sociedad irlandesa relacionados con su constitución y que requerían cierta revisión ${ }^{1}$.

\section{La necesidad de espacios sociales de deliberación}

La deliberación pública goza de mucho prestigio en el imaginario colectivo actual. Sin embargo, son pocas las ocasiones donde uno observa procesos constructivos de deliberación. Jornadas, congresos, platós, asambleas ciudadanas, son todos buenos espacios para la consultar acerca de los asuntos relacionados con el bien común. Estos espacios de reflexión y diálogo, con aspiraciones teórico prácticas, sobre formas efectivas de gobernanza, tanto locales, como nacionales e internacionales son especialmente pertinentes. Diferentes actores procedentes del mundo político, académico,

${ }^{1}$ La página web del gobierno de Irlanda donde se describe la naturaleza e historia del proyecto para reformar la Constitución es la siguiente: https://www.constitution.ie/Convention.aspx 
empresarial y civil comprometidos con la causa de la gobernanza y a quienes se puede observar como una red que contribuye al aprendizaje en evolución sobre esta área deberían poder reunirse para conversar en condiciones de igualdad. Los conceptos de «comunidades dialógicas» como grupos de personas comprometidos con una práctica que conversan en diferentes espacios sociales -revistas, congresos, jornadas-, «comunidades de discurso» o «comunidades de aprendizaje» nos sirven para ilustrar esta necesidad.

Desde otra perspectiva, estos espacios aspiran a contribuir, aunque modestamente, a la evolución del pensamiento social, actividad que cobra sentido en el contexto más amplio de individuos, comunidades e instituciones que se esfuerzan por contribuir al progreso social y al avance de la civilización.

Por último, y adoptando el sentido que le otorga el Instituto de Estudios en Prosperidad Global, los actores presentes en estos espacios pueden verse como integrantes de un discurso mundial en evolución sobre la gobernanza. Por discurso se significa el conjunto de conversaciones que actores comprometidos con una práctica -la gobernanza en este caso- mantienen en diferentes espacios con el propósito de mejorar esta práctica. A pesar de que las concepciones que puedan tener Habermas o Gadamer sobre estos conceptos nos son de utilidad, aquí se les pretende dar un sentido propio cercano a lo que se ha intentado explicar.

En definitiva, y tomando prestada la noción de Daniel Innerarity de innovación social, la pregunta axial que dirige la reflexión es: ¿cómo gobernar una sociedad que se ha vuelto mucho más compleja, inteligente, organizada y activa, que imbrica los ámbitos locales, nacionales e internacionales, que afronta problemas inusitados, y donde los instrumentos que se crearon para gobernarla parecen haberse quedado anticuados? El recientemente fallecido Ulrich Beck (1998) popularizó su caracterización de las sociedades modernas como sociedades del riesgo, aludiendo a unos cambios profundos en las formas de producción, las que ya no producirían tantos bienes como riesgos.

Cómo gobernar los riesgos globales (Solana y Innerarity, 2011), entonces, podría ser otra pregunta, formulada en términos diferentes, clave para avanzar hacia modelos de gobernanza más efectivos en un mundo en transformación.

\section{Sectores y niveles de gobernanza y preguntas marco}

\subsection{Gobernanza y gobierno formal}

El primer sector y el más obvio relacionado con la gobernanza es el gobierno formal y las instituciones u organizaciones públicas. En lo que respecta al Estado-nación, a pesar de que sigue siendo el actor principal de las políticas internacionales, muchos convendrían en decir que se ha desgastado en las últimas décadas, tanto por fuerzas propias internas como por fenómenos ajenos.

La globalización de los asuntos humanos, principalmente la economía, el arte, el conocimiento, la tecnología, la comunicación y el transporte -por no mencionar las amenazas y problemas más acuciantes, como el cambio climático, los movimientos 
poblacionales masivos, el desarrollo, el crimen transnacional o las armas de destrucción masiva- ha puesto en jaque al instrumento que mejor había respondido ante los asuntos públicos. Sin embargo, hoy día, a pesar de que éste tuviera los elementos considerados esenciales para el bueno gobierno, tales como el Estado de Derecho, la democracia representativa, la participación ciudadana, la rendición de cuentas, la transparencia y la conexión con los derechos humanos, no puede afrontar en solitario la misión para la cual fue ideado el Estado-nación. En palabras de Maltin Albrow, quien se atreve a afirmar que nos encontramos en otro momento histórico e incluso pretende caracterizarlo con el sello de «La edad global», la política no ha seguido el ritmo de globalización que han experimentado el resto de procesos sociales, algunos de los cuales ya hemos mencionado. Esto genera un déficit de gobernanza.

Otras instituciones públicas regionales e internacionales, ya sean militares o de gobierno, como la Organización de las Naciones Unidas, la Unión Africana, el Foro de Cooperación Económica Asia-Pacífico, la Organización de Estados Americanos, la Unión de Naciones Sudamericanas o el Consejo de Europa, buscan responder al déficit de gobernanza al que nos hemos referido. Sin embargo, y por muy alentadores que supongan estos avances, por sí solos no pueden responder efectivamente ante la monumental tarea que desafía a la humanidad en esta época.

La fuerzas internas que han desgastado el papel del Estado son de índole diferente aunque a todas les afectan esas dinámicas internacionales que se han señalado. Un problema acuciante que está azotando a los gobiernos nacionales es la creciente sensación de falta de legitimidad ante su ciudadanía. Antiguamente, la legitimidad la proporcionaban principalmente las elecciones democráticas en ambientes de libertades civiles y políticas, la creación de un contexto de bienestar social y económico para la mayor parte de la ciudadanía y la combinación de ambos. Sin minusvalorar estos dos elementos, progresivamente se han introducido otros fenómenos que incrementan la exigencia de los ciudadanos frente el gobierno: algunos exigen la estricta conexión del Estado con los derechos humanos, al punto que se ha llegado a decir que la legitimidad racional de un gobierno radica en su compromiso incondicional con el sistema de los derechos humanos; la necesidad de capital privado para sostener los programas de servicios sociales públicos, así como para financiar campañas políticas, ha descubierto la conexión existente entre el capital y el gobierno, sembrando dudas acerca de las motivaciones reales de ciertas decisiones políticas; la corrupción generalizada ha afectado la credibilidad de los gobernantes e incrementando la conciencia sobre la importancia de la ética pública y la transparencia; la profesionalización excesiva de los gobernantes y el alto salario de algunos han desprestigiado este servicio público; el cortoplacismo de las políticas, la competición excesiva entre partidos y el compromiso ante el electorado por encima de los principios ideológicos también han dañado la imagen de la política; el sometimiento de la política a las lógicas de los medios de comunicación; la falta de democracia interna en los partidos políticos; todo ello contribuye al aumento de la percepción de falta de legitimidad.

Tras estas reflexiones, brota la primera gran pregunta que posteriormente exploraremos en este artículo, y que otros autores abordarán en este libro, pero cuya respuesta no lograremos cerrar definitivamente: 
¿Qué innovaciones sociales podrían implementarse en el nivel del gobierno formal y las instituciones y organizaciones públicas, en los niveles local, nacional regional y global, para avanzar en términos de gobernanza y recobrar la legitimidad necesaria para el buen gobierno?

\subsection{Gobernanza y empresa privada}

El segundo sector relacionado con la gobernanza es el tejido empresarial. Aquí nos referiremos principalmente a las empresas privadas en lugar del sistema económico, debido a que su comportamiento es más fácil de analizar -no obstante, tendremos que referirnos a él someramente.

Las empresas privadas han adquirido tales dimensiones que su funcionamiento no afecta simplemente a intereses privados, sino que puede equilibrar y desequilibrar tanto gobiernos como sociedades. Las pequeñas empresas no tienen este impacto, sino las grandes corporaciones transnacionales que emplean, en muchas ocasiones, a cientos de miles de operarios - a veces en un solo país-, cuyos residuos pueden contribuir al daño ambiental y cuya inversión y capital puede condicionar el éxito de unas elecciones o dirigir las políticas públicas al haber comprado deuda pública a cambio de ciertas condiciones. Estas observaciones son ampliamente reconocidas hoy día, por lo que no ahondaremos demasiado en ello. Lo que más nos interesa es ver la relación entre la empresa y la gobernanza. Esta relación podríamos decir que tiene dos dimensiones que exploraremos a continuación: una interior y otra exterior.

La dimensión interior hace referencia a los esfuerzos por mejorar la gobernanza interna de la empresa. Principios tales como la democratización de la empresa, la disminución de la brecha entre el capital y el trabajo, la vinculación de las políticas de empresa con los derechos humanos, la responsabilidad social corporativa, la devolución de parte de las ganancias al entorno de donde provienen los beneficios, la incorporación de los trabajadores e incluso de los ciudadanos donde se ancla la empresa en los procesos de toma de decisiones, la participación de los empleados en los beneficios y pérdidas de la empresa sin contemplar despidos, por mencionar algunos, forman parte de la gobernanza empresarial. Estos principios expuestos contrastan con las formas más tradicionales de gestión empresarial, donde hay jerarquías claras, donde se busca exclusivamente la maximización de beneficios y la reducción de todo tipo de costes, donde los socios capitalistas y los trabajadores se mueven en esferas impermeables, etcétera. Aquí se abre toda una línea de acción y de reflexión relativa a cómo mejorar los procesos de gobernanza dentro de la empresa.

La segunda dimensión de la gobernanza empresarial, y que numerosos autores han tratado desde hace tiempo, se podría denominar externa ${ }^{2}$. Aquí se tratarían de abordar algunas cuestiones relacionadas con las planteadas en el punto anterior, tales como el

${ }^{2}$ Un libro ya clásico sobre este tema es el publicado por David Korten en 1995 Cuando las transnacionales gobiernen el mundo, Cuatro Vientos, Santiago de Chile, 1998. 
desafío de regular una economía mundial -especialmente la financiera- cuando los marcos regulatorios más potentes son nacionales. El incipiente derecho internacional privado, en ausencia de mecanismos internacionales de coerción -más allá de las sanciones que los Estados miembros de los tratados internacionales asumen voluntariamente tras resoluciones del Consejo de Seguridad principalmente-, parece insuficiente. Otra faceta relacionada es el papel de la empresa privada en promover ciertos servicios sociales por los que las instituciones públicas velan como la educación o la sanidad. Aquí se observa una necesidad incluso mayor de colaboración entre las instituciones públicas, las privadas y las organizaciones de la sociedad civil.

De aquí surgen otros interrogantes relativos a la gobernanza y la empresa: ¿qué tipo de gestión interna se puede implementar para que la empresa cumpla con su propósito de impartir bienes y servicios?, ¿cómo se pueden incorporar en términos prácticos algunos de los principios señalados en relación a la gobernanza interna de la empresa?, ¿cómo se pueden regular los mercados internacionales de una sociedad global configurada aún en clave de Estados nación?, ¿cómo equilibrar el propósito de la empresa de generar beneficios con su función de constituir un entorno para el desarrollo del potencial de sus miembros y con retornar a la sociedad parte de lo que se ha ganado?

En las siguientes secciones se volverá a algunas de estas cuestiones. Otros autores de la obra, también, abordarán esta cuestión de forma más directa.

\subsection{Gobernanza y sociedad civil}

La sociedad civil puede verse como un conglomerado de movimientos sociales, asociaciones, organizaciones no gubernamentales, familias y ciudadanos que interactúan entre sí, tanto como con las empresas privadas y las instituciones públicas. Una de las características del contexto social en el que surge el concepto de gobernanza es el deseo de la sociedad civil de tomar parte en los procesos políticos y en la gestión de la cosa pública sin necesidad de constituirse en forma de partido político. Esta ciudadanía, debido a factores de muy distinta índole, ha tomado mayor conciencia de su poder como colectivo y de las deficientes del orden económico, político y social, lo que ha hecho que se incrementen constantemente las demandas hacia los poderes públicos. Estas demandas, a pesar que en muchas ocasiones toman la forma de confrontación, se canalizan mayoritariamente mediante movilizaciones, manifestaciones y huelgas no violentas; sin embargo, en ocasiones no poco frecuentes, aludiendo a una ruptura del «contrato social» por parte del gobierno y de la empresa, la violencia también ha estado presente.

Un aspecto a tener en consideración es que en el imaginario colectivo persisten ideas románticas de lo que es la sociedad civil, el pueblo o los ciudadanos. Repetidamente, se evoca a la sociedad civil como la fuente de todo bien y a los poderes económicos y políticos como los males a combatir. Estos planteamientos, que están anclados en algunas teorías de la democracia, proceden de épocas en las que el poder era algo que se ejercía sobre la mayoría, ya fuera tiránica o ilustradamente, y poco contribuyen a una gobernanza efectiva de la sociedad compleja que hemos descrito y donde la colaboración y la reciprocidad parecerían ser irrenunciables. 
Otro aspecto incrustado en el imaginario colectivo, que dificulta la buena gobernanza y que encrespa la relación entre ciudadanos e instituciones públicas, es la narrativa que dice que cuando los poderes públicos no hacen bien su función, el pueblo se levanta para cambiar el orden de las cosas y reestablece la justicia y la paz. En los Estados que han experimentado dictaduras, el ideario suele resultar claro, avanzar hacia la democracia. Después, sin embargo, la evidencia ha mostrado que los problemas no se resuelven sólo con elecciones y que los intereses creados pueden manipular a la ciudadanía. Los colectivos que aspiran a tener un Estado propio, por otro lado, evocan ese futuro independiente como solución a todos sus problemas actuales -problemas causados por un opresor externo-, y dejan a un lado todo el resto de cuestiones fundamentales para la buena gobernanza, creyendo que se solucionarán en ese escenario de soberanía real posterior. Otros grupos, relacionados con cosmovisiones religiosas, a veces piensan que volviendo a la esencia de sus creencias y a los regímenes políticos que puedan alumbrar -aunque no sean compartidos por la mayoría de la población-, los males sociales que abundan en casi todos los países desparecerían. Dejando a un lado estos casos, diremos que, en las democracias consolidadas, este aspecto del imaginario colectivo tiene más dificultades aún, ya que tras la protesta y el levantamiento no hay un ideario, más allá de refinar los procedimientos democráticos y de combinar la democracia representativa con la directa.

Aunque es un hecho que la capacidad de la sociedad civil ha aumentado considerablemente -en parte gracia a la extensión de la educación formal- y que sus aspiraciones de tomar parte más activa en los procesos de gobernanza son genuinas, ésta no se halla libre de las fuerzas que afectan tanto a la política coma a la economía. La excesiva competencia, la búsqueda del interés personal, el apego a las ideas propias, la manipulación por los medios de comunicación, por los intereses económicos y por los lobbies, están también presentes ahí y, en ocasiones, con incluso más virulencia. En el caso que se relató anteriormente sobre la reforma de la constitución irlandesa, múltiples lobbies abordaron a los ciudadanos elegidos al azar para que asumieran, a cambio de primas, posturas cercanas a los intereses de esos grupos en las discusiones que tenían con los representantes políticos. Esto no parece ser un elemento aislado sino universal.

El profesor Michael Karlberg en su obra Beyond Conflict, muestra cómo el código cultural del normative adversarialism preponderante en los subsistemas sociales más importantes -la política, la economía y el sistema judicial-, ha calado muy hondo en otras esferas sociales, a saber: los medios de comunicación, la academia y los movimientos sociales ${ }^{3}$. Este código del que se deducen prácticas de confrontación como mejor estrategia para preservar los intereses propios y del grupo, parece afectar nega-

${ }^{3}$ Este código cultural se basa en unas concepciones de la naturaleza humana y de la sociedad que presentan al individuo como un ser egoísta que sólo busca maximizar sus intereses, y a la sociedad como un conjunto de grupos sociales que compiten entre sí. Estas concepciones, al afectar la forma en que se conciben los sistemas sociales y se articulan las prácticas, refuerza esas concepciones. Sin embargo, estas teorías normativas pasan por alto otros datos empíricos que muestran que los seres humanos no sólo se comportan egoístamente y que la colaboración es también una característica de la vida social (Kalberg, 2012: 200). 
tivamente a los movimientos sociales, cuyos ideales, por muy elevados que sean, terminan por diluirse porque la lucha con los poderes exteriores, finalmente se infiltra en el interior del movimiento, fragmentándolo e impidiendo que logren más victorias.

Por último, tal como ocurría con la empresa privada, existen múltiples organizaciones de la sociedad civil cuya labor se solapa con la de las instituciones públicas -y la de algunas empresas-, ya que han asumido la tarea de responder a ante algunas problemáticas que el gobierno no ha podido abordar con total eficacia, como, por ejemplo, el desarrollo comunitario, la educación, la protección del medio ambiente, los servicios de reinserción social, la alfabetización o la escolarización. De nuevo nos encontramos aquí con un espacio de intersección entre los tres sectores analizados: el gobierno, la empresa y la sociedad civil.

Con estas perspectivas en mente, se pueden vislumbrar diversas preguntas sin respuesta inmediata: ¿cómo evitar que el aumento del nivel de organización social y de la capacidad de la sociedad civil se atomice por las fuerzas de la competición y las luchas de poder características de otros ámbitos?, ¿qué papel constructivo pueden jugar los medios de comunicación más allá de criticar, de cara a generar una opinión pública bien informada, y cómo se han de gestionar para no caer presa, por un lado, de luchas ideológicas y, por otro, de intereses comerciales?, ¿qué papel habría de jugar la sociedad civil en la gobernanza?, ¿cómo equilibrar el deseo de la ciudadanía de participar en la toma de decisiones, con el aumento de responsabilidades que esto implica, las capacidades que exige y el tiempo que requiere?, ¿si la protesta no es el mecanismo más efectivo de construcción social, como encontrar modelos alternativos dentro de una sociedad dominada por la competición, el conflicto y la búsqueda del interés personal?, ¿qué capacidades se necesitan para iniciar un proceso de aprendizaje colectivo en un clima en el que la protesta dificulta la reflexión sobro los resultados de las decisiones a largo plazo? y ¿mediante qué tipo de programas educativos y procesos de socialización se pueden desarrollar dichas capacidades?

\subsection{Otros ángulos desde los que enfocar la gobernanza y preguntas finales}

A pesar de que aquí hemos explorado la gobernanza desde el papel del gobierno formal, de la sociedad civil y del mundo empresarial, la gobernanza se puede abordar desde otros ángulos también necesarios. Una aproximación muy útil es verla por niveles: gobernanza global, gobernanza regional, gobernanza nacional, gobernanza local y gobernanza multinivel. Otro enfoque resulta de conectar la gobernanza con subsistemas sociales: gobernanza económica, gobernanza del conocimiento, gobernanza de los riesgos, gobernanza energética, gobernanza de los derechos humanos...

Con independencia del ángulo desde el que nos aproximemos, una pregunta compleja apremiante que pretende conectar todo lo dicho en este capítulo, que durante la jornada guio la reflexión de todos los ponentes y que seguirá mostrándonos un sendero para el aprendizaje colectivo durante un largo período sería: ¿cómo gobernar la compleja red local, nacional e internacional, relativa al gobierno formal, las sociedad civil y el mundo de la empresa de forma que permita el avance hacia un civilización global más próspera, equitativa, inteligente y pacífica? 


\section{Explorando las implicaciones de algunos principios}

En esta sección se intentará iniciar una exploración en torno a las preguntas planteadas con anterioridad, por un lado, haciendo explícitos algunos supuestos y principios que nutren imperceptiblemente las prácticas dificultando los mecanismos de gobernanza, y, por otro, analizando las implicaciones prácticas de otra serie de principios. Los temas a tratar serán: la unidad y la justicia, el poder y la autoridad, los procesos de toma de decisiones, el papel del conocimiento y del aprendizaje, la mujer y la gobernanza, y la educación y la creación de capacidad.

\subsection{La unidad, la justicia y la colaboración}

La interconexión y la reciprocidad parece una de las propiedades inherentes del universo. En la realidad física, en los ecosistemas, en el cuerpo humano este principio es fundamental para la emergencia de la vida y de propiedades superiores a la suma de las partes. Los sistemas sociales también están interconectados, más aún en esta época. Existe cierto consenso, además, acerca de que, en una sociedad tan compleja como la actual, interconectada por todos los frentes, con procesos tan globalizados y con espacios para la toma de decisiones sin claras jerarquías, solapados y con múltiples intersecciones, se requieren grados de colaboración y de reciprocidad mucho mayores. Sin embargo, tal como hemos señalado previamente, la competición y la confrontación, sorprendentemente, siguen siendo las claves culturales dominantes en las que la población se socializa. Esta tendencia a la competición toma carices dramáticos claramente deleznables en las guerras y conflictos armados, pero también genera injusticias más sutilmente al estructurar la sociedad de tal forma que los grupos e individuos con más recursos o capacidades de partida terminen acumulando bienes desproporcionados. Esta misma tendencia hace que los sectores de la sociedad relacionados con la gobernanza -la sociedad civil, el gobierno o sector público y la empresa privada- se miren con recelo y desconfianza, entorpeciendo la necesidad de que colaboren. Esta tendencia, en última instancia, engendra actitudes egoístas que atomizan la sociedad en individuos o grupos sólo preocupados por su propio bienestar. Incluso en los ejemplos paradigmáticos de buena gobernanza, donde se observa al gobierno como configurador de espacios para que los grupos de interés dialoguen, esta tendencia a la competición se hace patente dificultando el encuentro de vías de actuación que beneficien a la mayoría.

En las relaciones internacionales también se observa una tensión entre dos realidades. Por un lado, el reconocimiento de que para enfrentar desafíos colectivos como el cambio climático, el desarrollo, las migraciones o el crimen organizado, los Estados han de colaborar. Por otro lado, la búsqueda del interés nacional como principio axial de las relaciones internacionales, impidiéndose así la acción colectiva. Este problema no es exclusivo de los gobiernos, sino que la ciudadanía y las empresas también parecen esperar de sus representantes políticos que persigan los intereses nacionales por encima de todo lo demás en su participación en los espacios supranacionales. En organismos públicos regionales e internacionales también se manifiesta esta paradoja, com- 
plicando la llegada a acuerdos. La soberanía nacional sin restricciones como eje vertebrador del orden internacional, a pesar de haber sido un instrumento que ha permitido el progreso durante siglos y que ha pacificado significativamente las relaciones internacionales, parece haber agotado su funcionalidad para un mundo globalizado.

Otra implicación de la unidad y de la justicia para la gobernanza está relacionada con el papel de los grupos minoritarios oprimidos históricamente, ya sea dentro de un territorio nacional o del orden internacional. Un paso importante dentro algunos países ha sido darles mayor protagonismo en los procesos sociales y políticos. Algunos han desarrollado innovaciones sociales como las cuotas de representación o la discriminación positiva. Sin embargo, si nos aproximamos a este fenómeno con la visión de contribuir al avance de la civilización, no veríamos la incorporación de estos grupos a ciertas esferas de poder como un fin en sí mismo, y mucho menos como una estrategia para imponerse sobre otros grupos -invirtiendo los roles de víctima y opresor-, sino como una oportunidad para realzar la capacidad de este grupo para contribuir su parte única al enriquecimiento colectivo. Cada pueblo, cada nación, tiene algo singular que ofrecer al jardín humano, y el desafío mayor es que todos, en igualdad de oportunidades, puedan aportar a la construcción de una sociedad unificada, próspera y justa para todos, en la que lo global y lo local se complementan y armonizan.

En resumen, a no ser que se cuestionen algunas de las concepciones acerca de la naturaleza de la sociedad, en la que se ven individuos compitiendo por escasos recursos o grupos con intereses irreconciliables negociando por las alternativas «menos malas» para todos, difícilmente se podrá avanzar hacia una sociedad próspera, equitativa y pacífica. Este cuestionamiento que se sugiere no busca adoptar ciegamente una concepción ilusa de la naturaleza humana y de la sociedad como entidades que funcionan con plena armonía, sino que invitaría a explorar empíricamente el comportamiento humano, tanto individual como colectivo, para tener una visión más objetiva de las diferentes inclinaciones que lo distinguen y de las potencialidades que se podrían cultivar mediante la educación y la socialización para responder ante la creciente mundialización, complejización e interconexión de los desafíos de la época en que vivimos.

La humilde pero creciente experiencia de la comunidad bahá'í, un microcosmos de 7 millones de personas representando todas las razas, etnias, naciones y culturas del mundo, muestra que es posible articular la organización social alrededor de los principios de unidad en diversidad, de justicia social y de reciprocidad. Su experiencia demuestra que, a pesar de que la búsqueda del interés personal o nacional son tendencias a tener en consideración, éstas se pueden supeditar al bienestar colectivo y al internacional. La manera en que equilibra las autonomías locales y nacionales dentro de un marco global bajo el liderazgo de institución internacional legítima y democráticamente elegida por representantes de todos los países del mundo, es un buen recordatorio de que las analogías del cuerpo humano y del cuerpo social son aplicables a las relaciones humanas. La diversidad, no obstante, no se sofoca en aras de la unidad, sino que se considera un imperativo para la existencia de unidad y, por ello, incluso se promueve. Todavía, no obstante, son muchos los desafíos que tendrá que ir enfrentando para lograr suponer un modelo alternativo de organización social, pero la experiencia de 172 años de existencia parece prometedora. 


\subsection{El poder y la autoridad}

Hablar de gobernanza nos remite ineludiblemente a la cuestión del poder y la autoridad, conceptos que suelen estar interconectados. Sin embargo, las concepciones predominantes del poder, especialmente, se refieren a la capacidad de modificar el comportamiento y/o las conciencias en la dirección en la que el que lo ostenta considera oportuno. Por ello, el poder se suele asociar con dominación, subordinación e incluso manipulación.

Como se podrá observar, con estas concepciones de poder difícilmente se podrán articular mecanismos efectivos de gobernanza en una sociedad mundializada, con múltiples sectores entrelazados, con espacios para la toma de decisiones que se solapan o con unas dosis de incertidumbre inusitadas.

Para poder mejorar las estructuras, procesos e instrumentos de gobernanza en todos los niveles, se requieren dosis de poder no liberadas hasta la fecha. En este sentido, las concepciones tradicionales que veían al poder como un centro divino en la tierra que Dios otorga al emperador absoluto, los planteamientos modernos que aspiran a llegar racionalmente a un espacio superior desde el que dirigir la sociedad hacia la emancipación, las visiones posmodernas que ven el poder esparcido por todas partes y a todas las personas luchando por él, u otras perspectivas más sofisticadas que ya sea que exploren otras formas de dominación mediante la construcción social del sentido común por parte de las clases dominantes o que analicen la relación entre el poder y el conocimiento, no son suficientes para describir el funcionamiento del poder ni pueden servir de fundamento sobre el que asentar una sociedad que requiere de cuotas mayores del mismo para transformar sus relaciones en función del principio de la unidad orgánica de la humanidad.

Además de las formas descritas en las que actúa el poder, algunos autores introducen nuevas percepciones que enriquecen la comprensión e indican avenidas preliminares prometedoras. La comunidad bahá'í, un caso al que recurriremos en ocasiones por llevar más de un siglo aprendiendo acerca de la aplicación de algunos de los principios enunciados aquí en su gobernanza interna, también parece sostener varias de las concepciones que se van a señalar.

Talcott Parsons observa el poder como algo sinérgico, como la capacidad de generar consensos colectivos para dar dirección a la sociedad de tal modo que se beneficie a la mayoría. Cuando individuos, comunidades e instituciones colaboran para mejorar la gobernanza de la sociedad, parece que se liberan dosis de poder que permiten logros imposibles de otro modo. Esta es la razón por la que grupos pequeños, cuando trabajan de forma armoniosa, han conseguido grandes victorias. El poder, aquí, se utiliza para «actuar con» no para «actuar sobre».

En el nivel colectivo, se podría decir, además de lo expuesto sobre la colaboración, que cuando las instituciones y las comunidades logran equilibrar los talentos, encontrar consensos, tratar con justicia los temas que abordan, se canalizan poderes insólitos que permiten de nuevo logros imposibles de lograr mediante la competición y la lucha exclusivamente.

Otra forma de abordar el poder, tal como menciona el sociólogo Anthony Giddens, es en términos de capacidades. El poder se libera en la acción de los individuos prin- 
cipalmente. Este poder está relacionado con el conocimiento que se tiene, con el conjunto de recursos intelectuales, emocionales y espirituales que posee el individuo, con la pureza con la que se actúa, con la verdad que porta lo que dice, con la humildad con que se profieren las palabras, con la belleza de la causa que defiende o con la rectitud de conducta del que actúa.

Otro elemento relacionado con el poder y con la autoridad se encuentra en los estilos de liderazgo. La autoridad moral de las instituciones, quizás, recaiga principalmente en la legitimidad que les otorga la forma en que ejercen su liderazgo. No es menester hacer una incursión profunda en esta cuestión; baste con decir que el liderazgo autoritario - tan inclinado a la imposición-, el paternalista -que impide el desarrollo de las capacidades de aquellos a quienes sirve-, el manipulador -que busca su propio interés o imponer su visión mediante técnicas de dominación más sutiles-, e incluso el democrático - a veces devenido en demagogia por responder ante su electorado con vistas a la reelección- parecerían también insuficientes. Un estilo de liderazgo conectado escrupulosamente con la ética, orientado hacia el servicio y bien común, sin intereses particulares y consciente de que trabajar por el bien social es la causa más elevada a la que se puede aspirar, parece el más apropiado para guiar, ya sea desde el gobierno formal, desde la empresa o desde la sociedad civil, los procesos y estructuras relacionados con la gobernanza.

Por último, ligado también a la cuestión del poder y la autoridad, está la forma en que se elige a los representantes públicos, mecanismo que se suele imitar en otras esferas sociales. La teoría de la democracia aboga por combinar, al menos, tres componentes: representantes elegidos, espacios de participación ciudadana y movimientos sociales. La idea relacionada con las concepciones dominantes de poder que hemos analizado, la de que cuando se llega al poder se intenta imponer la visión frente al resto, ha hecho que se estructure el gobierno en contrapesos de poder: gobierno y oposición, políticos y movimientos ciudadanos, debate parlamentario y debate ciudadano crítico, comunicación política y supervisión de los medios de comunicación.

El aspecto para el que se quiere proporcionar otros modelos es de las elecciones. Actualmente los representantes públicos se eligen de entre los candidatos por partidos políticos que supuestamente reflejan las diferentes sensibilidades ciudadanas. Sin embargo, hay una serie de problemas conocidos que impiden una buena gobernanza. La dependencia de campañas financiadas por las empresas o inversores para ganar las elecciones que hace que ganen los más pudientes y no siempre los más capaces, la profesionalización de dirigentes de los partidos, la respuesta ante el electorado y el apego al poder que conducen a adoptar decisiones electoralistas son algunos de ellos. Las asambleas populares, donde pueden participar todos los ciudadanos que quieren toman decisiones colectivas, también tienen serias dificultades, ya que a veces los menos prudentes y los más insistentes son los que imponen sus ideas ante la mayoría. Además, este mecanismo dificulta la profundización en ciertas cuestiones que requieren conocimiento más profundo y atención sosegada.

La experiencia de la comunidad bahá'í con sus procesos electorales -un microcosmos modesto, aunque de alcance mundial con aproximadamente siete millones de personas procedentes de 157 países-, puede ilustrarnos acerca de otras formas alternativas de elección de representantes. Algunos de sus rasgos más sobresalientes son los siguien- 
tes. En una zona geográfica pequeña -una localidad-, con unas condiciones previas necesarias -como ciertos procesos comunitarios que permitan que la gente interactúe y se conozca y cierto compromiso con los principios que guían el proceso electoral-, una vez al año todos los bahá'ís mayores de 21 años participan en una elección que consideran sagrada y en la que todos pueden ser elegidos. No existen postulados, candidaturas ni campañas. Por medio de una reunión presencial a la que son convocados, tras un periodo de reflexión, oración y meditación, cada participante, en secreto, anota a las nueve personas cuyas cualidades y capacidades considera más deseables para dirigir los asuntos de la comunidad y guiar su progreso. Las personas que consiguen más votos, conforman el Consejo de Gobierno Local -la Asamblea Espiritual Local- por un año y, salvo motivos mayores, tienen el deber moral de desempeñar esta función, sin dedicarse profesionalmente a ello. Como individuos no tienen una posición especial dentro de la comunidad, pero el cuerpo elegido es la autoridad moral de la zona y sus decisiones, que sólo pueden guiarse por el interés general, son acatadas por el conjunto del electorado. La motivación detrás de su desempeño no es la de una oportunidad para imponer su visión particular al resto sino el espíritu de servicio desinteresado. Este proceso, cuando hay demasiadas personas en una localidad, se realiza en dos fases, una primera donde se escogen representantes por sectores que actuarán como delegados en una elección local donde podrán elegir no sólo de entre los delegados, sino de entre todos los electores. En el nivel nacional, también realizan un proceso similar para elegir a su cuerpo de gobierno nacional. En este caso, se efectúan elecciones regionales donde se escogen delegados que participarán más adelante en una Convención Nacional para elegir al Consejo de Gobierno Nacional. Los delegados no son candidatos, sino que pueden elegir de entre todos los electores del territorio nacional. En el plano internacional, por último, cada cinco años, los delegados nacionales, que son los nueve miembros de los cuerpos de gobierno nacionales, se reúnen en una Convención Internacional para escoger a su cuerpo de gobierno supremo, la Casa Universal de Justicia. Con independencia de los niveles, los principios destacados son exactamente los mismos, y su viabilidad ha sido probada durante más de un siglo y especialmente desde 1963, cuando se eligió por primera vez la Casa Universal de Justicia.

\subsection{Los procesos de toma de decisiones}

Si se aspira a avanzar en términos de gobernanza y a tomar en serio el papel del gobierno como configurador de espacios de interacción para que los actores involucrados en ciertos temas lleguen a acuerdos y encuentren vías de actuación colectivas, se le ha de prestar especial atención a cómo se toman las decisiones. Los procesos de toma de decisiones son un aspecto vital de la gobernanza a todos los niveles.

En las secciones anteriores ha quedado manifiesto que los mecanismos de dominación y manipulación habrían de dar paso a formas más cooperativas de funcionamiento. Sin embargo, debido al énfasis que se le ha dado a la competición, a la confrontación y a lucha -no violenta-, son pocos los sectores de la sociedad que pueden conversar dejando a un lado la búsqueda frenética de su interés personal o grupal. Incluso individuos y colectivos bienintencionados experimentan dificultades cuando se 
enfrentan a la tarea de dialogar con otras personas o grupos para generar entendimientos colectivos y actuar con unidad de visión. En las relaciones internacionales, en los procesos de resolución de conflictos, en los espacios locales donde hay grupos «negociando», los procesos de toma de decisión se dan por oposición de ideas, retardando mucho los acuerdos. Así, los modelos convencionales de discusión y debate, de confrontación de ideas, que se observan en los medios, en la academia o en las sesiones parlamentarias, y que a menudo son impulsados por el ego, la ideología o la competencia de grupos de interés, han resultado ser insuficientes para los complejos desafíos que enfrenta la humanidad. Claramente se requieren modelos más competentes. A este respecto, el Instituto de Estudios en Prosperidad Global señalaba:

- Los procesos maduros y eficaces para la toma colectiva de decisiones requieren que la investigación de problemas complejos sea sincera, sistemática y colaborativa. Tales procesos se enriquecen con la participación de personas provenientes de diversos orígenes, perspectivas y puntos de vista, en un espíritu de servicio al bien común, con una postura humilde de desprendimiento de sus propias ideas preconcebidas. En este contexto, la unidad, la imparcialidad y el juicio maduro pueden lograrse mejor si la toma de decisiones es guiada por la identificación y aplicación de principios morales o espirituales -tales como los principios de justicia y fideicomiso colectivo que se han analizado anteriormente. Por otra parte, estos procesos también deben ser receptivos a los insumos y la retroalimentación de la comunidad en general, y los procesos deberán ser protegidos de la manipulación y presiones de grupos de interés poderosos y egoístas.

- Los procesos de toma de decisiones que se caracterizan por estos rasgos permiten que las organizaciones y comunidades logren coherencia y enfoque, formulen objetivos comunes, gestionen los recursos colectivos y promuevan estrategias de desarrollo, a fin de movilizar diversos talentos y capacidades, y fomentar el espíritu de iniciativa y empresa. Sin embargo, estos procesos de toma de decisiones no se logran fácilmente en la práctica y requieren paciencia, sensatez y una perspectiva de desarrollo para ser alcanzados. ¿Pero cómo se puede fomentar y desarrollar esta paciencia, sensatez y perspectiva en el ámbito de la gobernabilidad? ${ }^{4}$

La experiencia de la comunidad bahá'í ofrece de nuevo algunas perspectivas interesantes, ya que lleva años aprendiendo a utilizar un modelo colectivo para la toma de decisiones alternativo, unificador y constructivo, que se denomina consulta, y que, aunque a primera vista pudiera parecerse a otros modelos como el de la acción comunicativa de Habermas, tiene sus propias particularidades que han demostrado cierta eficacia en la práctica. Este último aspecto es relevante. Mientras que autores como Rawls o Habermas plantean modelos teóricos alternativos para el debate público y la toma colectiva de decisiones, éstos no han mostrado su funcionamiento en la acción.

${ }^{4}$ Instituto de Estudios en Prosperidad Global, Reflexiones sobre la gobernabilidad, julio 2012, p. 4. En: http://www.globalprosperity.org/documents/ISGP_Reflections_on_Governance.pdf 
El modelo de la comunidad bahá'í, no obstante, lleva mostrándose funcional más de 100 años.

En el proceso de consulta, evitando los debates y las polémicas, los participantes solicitan la opinión de los demás en un ambiente de respeto mutuo de investigación colectiva, basándose en los supuestos de que la realidad es una, aunque compleja y multifacética, y de que los diversos puntos de vista pueden ser complementarios y ampliar el entendimiento colectivo de un tema. Los participantes también tienen que cumplir ciertas condiciones, así que no sólo es un procedimiento, ya que se esfuerzan por ofrecer sus propios puntos de vista con humildad y con desprendimiento de las ideas de partida, reconociendo que las aportaciones que ha hecho cada uno ya no son suyas, sino que forman parte del grupo para ser aceptadas, rechazadas, modificadas o complementadas. Cada idea se analiza detenidamente, con mirada crítica y de manera franca y abierta. Cada persona, cuando se expresa, lo hace libremente, pero sin menospreciar la idea del otro ni insistir excesivamente, y teniendo el tacto necesario para no herir al resto. De este modo, se intenta clarificar lo máximo posible el tema de consulta y los participantes también tratan de buscar los principios subyacentes que han de guiar la resolución final, teniendo en cuenta que lo que se busca es un acuerdo que promueva el bienestar de todos a quienes sirven. Tras explorar el tema, exponer las ideas, documentar y fundamentar las diferentes posturas, se esfuerzan por alcanzar consensos. Si no se logra, prevalece la voz de la mayoría y, posteriormente, todos están moralmente compelidos a actuar en la línea acordada, con independencia de la idea de origen, ya que se considera que es la puesta en práctica del acuerdo por parte de todos y el tiempo los que determinarán si la decisión era apropiada o no. De no serlo, al haber actuado en unidad, podrán reflexionar y explorar los aprendizajes suscitados, introduciendo posteriormente este aprendizaje en la toma de decisiones.

Como se ha mencionado, la comunidad bahá'í continúa aprendiendo a aplicar este modelo, pero ya ha demostrado ser muy eficaz en diversos contextos, tanto formales como informales. Es empleado universalmente en las instituciones de gobierno de la comunidad bahá'í, en sus comités y agencias. También se utiliza ampliamente en las familias, los negocios, las organizaciones sin ánimo de lucro y otras actividades bahá'ís. Por otra parte, el hecho de que se esté dando con eficacia en prácticamente todos los contextos culturales del mundo, muestra que es un modelo alternativo de toma de decisiones que podría trasladarse a otros ámbitos más amplios. De hacerse así, para que sea eficaz, los individuos e instituciones que intentaran aplicar este mecanismo, tendrían que tener cierto compromiso con los principios enunciados, tanto los individuales -de respeto, humildad, escucha, desprendimiento, libertad, claridad, tacto-, como los procedimentales. En este sentido, un proceso de socialización previo parecería oportuno, ya que sólo se logrará refinar su funcionamiento en la práctica regular y continuada.

\subsection{El papel del conocimiento y del aprendizaje}

Una de las caracterizaciones más generalizadas que algunos pensadores contemporáneos hacen y que pretende describir el cambio social que parece estar ocurriendo en las sociedades post industriales es la de «sociedades del conocimiento». Sin embargo, afir- 
mar que el conocimiento es el eje de vida social puede verse a la vez como algo descriptivo y normativo, como lo que está siendo y lo que debería ser. Aquí nos centraremos en el aspecto normativo, ya que si la descripción que se ha hecho de las transformaciones que han acontecido es acertada, avanzar en términos de gobernanza implica avanzar muy notoriamente en términos de conocimiento.

Si se han de establecer las estructuras y los procesos de gobernanza tan complejos que se han apuntado, abordar la cuestión en modo de aprendizaje parece vital. Además de una actitud, esto supone un enfoque metodológico particular, ya que el conocimiento que se ha de generar acerca de las estructuras y procesos de gobernanza es un conocimiento práctico, no generado aún, y que ha de ir emergiendo de los esfuerzos crecientes de individuos, comunidades e instituciones por ponerlos en práctica. Este enfoque requiere que los actores comprometidos con la acción, señalados anteriormente, desarrollen ciertas capacidades, entre las que descuellan la capacidad de definir problemas y áreas de aprendizaje prioritarias sin fragmentar la aproximación a la realidad social, la capacidad de reflexionar constantemente sobre la acción, la capacidad de actuar y reflexionar colectivamente, la capacidad de consultar y de llegar a acuerdos concernientes a lo que beneficia a la mayoría, la capacidad de recurrir tanto a la ciencia como a los principios rectores que deliberadamente se pretende que dirijan las relaciones humanas en una búsqueda sin tregua pero sosegada de modelos alternativos que demuestren su efectividad en la acción, la capacidad de revisar y ajustar constantemente las decisiones y los resultados de los planes y enfoques adoptados una vez se ha dejado tiempo suficiente para observar sus consecuencias y la capacidad de incorporar a largo plazo todo este conocimiento práctico acumulado en los procesos de toma de decisiones en todos los niveles.

Hay que reconocer que lo declarado en el párrafo anterior no es fácil y que existen ciertas tendencias que dificultan el proceso de aprendizaje colectivo necesario para la gobernanza al que se ha aludido. Identificar estas tendencias, sin recrearse en ellas, puede resultar útil de cara a superarlas. Una de ellas es la lucha de poder, los cambios de programa con cada gobierno, la planificación cortoplacista, la constante confrontación y la política partidista que no sólo afecta al gobierno formal, sino que se filtra en todas las esferas sociales. A esto se le suma la falta de credibilidad de las instituciones dotadas de autoridad, algo que requiere su propio abordaje. Esta tendencia a la confrontación se manifiesta, como ya hemos dicho, en la relación entre los movimientos y asociaciones civiles y el gobierno, o entre el gobierno y la empresa, o entre la empresa y la sociedad civil. En este clima, reflexionar sobre las decisiones y acciones que requieren medio plazo resulta algo extremadamente complicado. La interpretación de lo que acontece desde marcos ideológicos muy cerrados, también impide leer la realidad acertadamente, ya que lo que se busca es la justificación en lugar del aprendizaje real. Reemplazar los marcos ideológicos -a veces implícitos- por marcos de análisis más amplios que permitan la evolución de la comprensión, pero sin renunciar a que se sostengan sobre ciertos principios explicitados desde los que se explora la realidad, parece muy oportuno. La sensación de riesgo y amenaza tan extendida en muchas sociedades no suele favorecer tampoco los procesos de aprendizaje ni la correcta toma de decisiones. Tal como Cass Sunstein demuestra, cuando la toma de decisiones acerca de temas públicos se produce en un clima de riesgo generalizado, el análisis de los 
costos-beneficios no suele ser efectivo, produciéndose, en ocasiones, debido al tipo de respuestas que se idean ante situación de amenaza percibida, problemas mayores de los que se pretendían abordar (Sunstein, 2006). Por último, y sin ánimo de ser comprehensivo, habría que destacar la dificultad que supone la tendencia a abordar las cosas de forma simplista, fragmentada y en pares opuestos, tendencia reforzada además por los medios de comunicación. Un proceso de aprendizaje colectivo exige abordar cuestiones complejas de forma sistémica y profunda, dejando cierto margen para la incertidumbre y la ambigüedad, ya que la complejidad de la sociedad hace que no haya fórmulas claras que reproducir.

El último elemento a señalar tiene que ver con los niveles de aprendizaje y el flujo del mismo. La gobernanza abarca muchos ámbitos y se da en muy distintos niveles. Si lo vemos desde el prisma de la gestión de los asuntos públicos, tenemos ineludiblemente que tratar la cuestión de los centros de autoridad legítima donde el aprendizaje también ha de darse. Estos centros, aunque desprestigiados por las razones expuestas, están bastante definidos en el ámbito local y nacional, pero menos en el internacional. La coordinación de los niveles locales, nacionales, internacionales e intersectoriales parece ser otro desafío ingente, más todavía cuando en los niveles supranacionales existen tantos espacios para la toma de decisiones y donde ninguno de los cuales puede arrogarse ser la autoridad legítima. La necesidad de coordinación, colaboración, reciprocidad e interconexión, por tanto, se hace más patente aquí que en ningún otro lado.

\subsection{La mujer y la gobernanza}

Es difícil subrayar tanto como merece la importancia que tiene la incorporación de la mujer a todas las esferas sociales para avanzar hacia una sociedad donde las relaciones humanas estén caracterizadas por la cooperación, la reciprocidad y el apoyo mutuo. La gobernanza es quizá uno de los ámbitos más importantes donde este fenómeno habría de darse para lograr un progreso significativo.

Es un hecho consabido que la mujer ha estado históricamente excluida de la vida pública y, a pesar de que durante las últimas décadas su incorporación se ha promovido desde muchos frentes y de que se han creado mecanismos para acelerarla, esta incorporación aún es insuficiente.

Existen dos aspectos de esta incorporación que merecen ser destacados relacionados con la gobernanza: el propósito y repercusiones de la incorporación de la mujer a las estructuras y mecanismos de la gobernanza y su presencia en los espacios de toma de decisiones.

Algunas corrientes feministas destacan la diferencia entre la incorporación de la mujer a espacios otrora dominados por los hombres y la adopción de ciertos comportamientos masculinos para estar en esos espacios. Sírvanos de ejemplo la política y la economía. En ambas esferas sociales, el domino masculino ha hecho que la competición, la fuerza, individualismo, la agresividad del carácter e incluso la insensibilidad caractericen el desempeño de funciones políticas y económicas. En cierto sentido, acceder a esos espacios pasa por adoptar esas mismas cualidades y comportamientos. Las mujeres, por tanto, para poder acceder, también han tenido que desarrollarlas. 
¿Pero qué ocurre cuando esas mismas cualidades y principios rectores del comportamiento se tornan insuficientes para afrontar los desafíos de gobernanza de una sociedad compleja, global e interconectada? Es aquí donde los análisis del comportamiento histórico de las mujeres cobran mayor sentido. Ya sea por diferencias biológicas o por un larguísimo proceso de socialización desde los primeros grupos humanos hasta nuestros días, parece que las mujeres han desarrollado más la empatía, la capacidad de llegar a consensos, de trabajar en equipo y de utilizar el poder de maneras cooperativas. También han logrado desarrollar otras formas de racionalidad y de inteligencia que van más allá de la simple búsqueda instrumental de beneficios y de minimización de pérdidas, teniendo en cuenta otros aspectos más sutiles pero fundamentales y percatándose de otras sensibilidades, aspectos sumamente necesarios para lograr el bien común. Esas cualidades y principios, como vimos al inicio, parecen corresponderse con los cambios en los principios organizativos que la política debía experimentar para adaptarse a un mundo entrelazado y global. La línea que se desprende de todo esto, entonces es doble: el acceso de la mujer a todos los espacios sociales y la difusión de ciertas cualidades vitales históricamente relacionadas con el comportamiento femenino.

Un tema relacionado con el anterior es el papel que la mujer puede desempeñar en el mantenimiento de la paz. Hasta la fecha, los espacios donde se tomaban las decisiones acerca de la pertinencia de hacer la guerra eran exclusivos de hombres. La tendencia parece haber cambiado un poco, aunque todavía el porcentaje de mujeres en ellos es minúsculo. Si los planteamientos anteriores son ciertos, y si se tiene en cuenta que la mujer históricamente ha desempeñado un papel más destacado en la educación de las nuevas generaciones que los hombres, la utilización de medios diplomáticos y pacíficos en los conflictos sociales se incrementaría sustancialmente con la incorporación de las mujeres a esos procesos. Tanto por sus capacidades de llegar a consensos, de cooperar, de trabajar en equipo, de mirar la realidad holísticamente, como por la mayor sensibilidad que ha podido suscitar el hecho de ser madre, la mujer puede tener un papel singular en el establecimiento de la paz.

Un último comentario sobre la incorporación de la mujer a todas las esferas de la vida social y, en particular, a los espacios que tradicionalmente se asocian con el poder. La sociedad en que vivimos enfrenta muchos desafíos, uno de los cuales y de los más sobresalientes, sin duda, es el tratado aquí: su gobernanza. Por ello, la incorporación de la mujer en plenitud a la vida social, política y económica, vendría bien en verse desde la óptica de estar en igualdad de condiciones con el hombre para trabajar en colaboración en pos de un mundo mejor para todos. Los planteamientos divisorios a veces relacionados con los movimientos de emancipación de las mujeres o con los programas de empoderamiento femeninos no tienen cabida. Crear una sociedad global, unida, pacífica, justa y próspera exige que todos los segmentos de la sociedad -el $50 \%$ de las cuales son mujeres- puedan trabajar por ese fin.

\subsection{La educación y la creación de capacidad}

Avanzar hacia las perspectivas sugeridas en las secciones anteriores requiere atender tanto a los arreglos institucionales y organizativos como a las capacidades de los indi- 
viduos que conforman dichas instituciones y organizaciones. Existe una relación bidireccional entre estos dos aspectos -los individuos y las estructuras sociales-, se afectan mutuamente $y$, por tanto, para lograr cambios significativos en materia de gobernanza, se les debería prestar atención a ambos. En esta sección vamos a centrarnos en el proceso necesario para que los individuos puedan desarrollar ciertas capacidades relacionadas con las gobernanza. La confianza en el poder de la educación para moldear el carácter y liberar las potencialidades de los individuos se fundamenta en la concepción de que el ser humano no es inevitablemente egoísta, competitivo y agresivo. Se reconoce que existen esas inclinaciones -que se fortalecen además en un proceso de socialización donde las estructuras y los sistemas las refuerzan y recompensan-, pero también se constata la evidencia empírica de que existe la tendencia a la cooperación y al altruismo. El entorno cultural, incluyendo la educación, favorece que se desarrollen y expresen más plenamente unos aspectos de la condición humana u otros.

A lo largo del artículo, pero especialmente en las últimas secciones, se han destacado varias capacidades necesarias para la buena gobernanza que muy difícilmente se podrán desarrollar si, desde la infancia, no se abordan dentro de programas educativos. En ese período, la honradez, el altruismo, el apoyo mutuo, la sinceridad y la humidad son más fáciles de desarrollar debido a la mayor maleabilidad del carácter humano. Existe acuerdo en que durante la adolescencia el individuo adopta los principios y elabora las concepciones que le guiarán prácticamente durante el resto de su vida. Por ello, algunos aspectos relativos a la gobernanza serían más fácil de adoptar si se introdujeran en esa franja, a saber: el compromiso social, el trabajo en equipo, la importancia de la interconexión, las concepciones de poder, el papel de la unidad y de la justicia, la importancia del servicio al bien común, los estilos de liderazgo, la capacidad de auto-expresión, la capacidad de escuchar a los demás, la capacidad de tener en consideración las opiniones e ideas de los que históricamente no han tenido voz alguna, la capacidad de suspender los juicios preconcebidos propios y de volverse hacia nuevas perspectivas con mente abierta, la capacidad de ver la diversidad como fuente de riqueza y fuerza, la capacidad de aplicar métodos de investigación sistemática a la investigación de temas o problemas complejos, y la capacidad de elevar el discurso al nivel de principios morales o espirituales para luego dejarse guiar por tales principios en la formulación de las decisiones.

Otros aspectos que los programas de educación deberían abordar en relación a las estructuras y procesos de gobernanza, muchos de los cuales se señalaron arriba, serían los procedimientos electorales descritos, las cualidades necesarias para servir en cuerpos de gobierno elegidos, cómo operar en un modo de aprendizaje, el papel de la ciencia, su naturaleza y funcionamiento, las cualidades y procedimientos relativos a la consulta y a los procesos de toma de decisión, el papel de la mujer en la vida social, por mencionar algunos. Otros aspectos los mencionaba el Instituto de Estudios en Prosperidad Global en su documento sobre gobernanza:

- Además, hay otros requisitos de la gobernabilidad eficaz que pueden ser fomentados mediante la capacitación y la educación, consistentes en un espíritu de apertura e inclusión en las relaciones que establecemos con los demás; una ética de trabajo que se caracteriza por el espíritu de servicio al bien común, 
y una actitud de paciencia, flexibilidad, y resiliencia frente a las dificultades y reveses. Por otro lado, los enfoques de la gobernabilidad que se unifican y potencian mutuamente solo se pueden desarrollar cuando las personas pueden crear un clima de confianza y respeto mutuo, libres de hábitos nocivos, como la murmuración, que envenenan la atmósfera dentro de las organizaciones, instituciones y comunidades. Nuevamente, ¿cómo pueden los programas de educación y capacitación de esta índole ser desarrollados y aplicados, en una escala creciente, de manera constructiva, incluyente y atractiva?

- Tal como se indica en este párrafo, las pregunta apremiantes que brotan de estas reflexiones son el cómo introducir todos estos aspectos en programas formales e informales y otros mecanismos de socialización que pudieran educar a las nuevas y presentes generaciones en todos estas cuestiones relativas a la gobernanza, y cuáles serían los mejores enfoques metodológico para que contingentes crecientes de personas pudieran desarrollarlas.

\section{Conclusiones}

Este artículo puede verse como una invitación al diálogo colectivo en torno a la cuestión de la gobernanza. Su planteamiento refleja cierto entendimiento en evolución y plantea avenidas específicas para la indagación futura. Al mismo tiempo, recoge las conversaciones mantenidas entre múltiples actores durante los últimos meses, conversaciones que llegaron a un clímax en la jornada sobre gobernanza celebrada en mayo en el Centro de Estudios Políticos y Constitucionales y que constituye la apertura de un espacio social donde, desde diferentes ángulos, se seguirá explorando la cuestión de la gobernanza. Mediante ciclos de seminarios, publicaciones, visitas, conferencias, simposios, jornadas y mesas de trabajo que aglutinan a representantes de la sociedad civil, del mundo académico, de la empresa y del gobierno comprometidos con la causa de la gobernanza, se pretende intensificar la reflexión colectiva sobre esta temática. Algunas conclusiones provisionales de lo mencionado en este estudio preliminar se detallarán a continuación.

La sociedad moderna ha experimentado profundas transformaciones, por lo que los instrumentos políticos que se habían diseñado para gobernarla han quedado obsoletos. La capacidad de análisis de dichas transformaciones desde la política no está a la altura de las circunstancias, así que, al fallar los diagnósticos, las prescripciones no son las acertadas. La globalización ha impuesto una complejidad añadida a la gestión de lo público, ya que se carecen de espacios lo suficientemente legitimados y con poder efectivo para la toma y ejecución de decisiones. La complejidad creciente exige modos de actuación distintos. Entre ellos, destaca la necesidad de colaboración entre la sociedad civil, el sector empresarial y el sector público. Sin embargo, las lógicas dominantes propician la competencia en todas las esferas sociales, complicando sobremanera la necesidad de colaboración. Se requiere poner el foco en iniciativas emergentes, aunque minoritarias, de acción y organización social basadas en principios tales como la colaboración y la reciprocidad, la justicia social, el equilibrio entre la centralización y la descentralización, la sostenibilidad y la subordinación del interés individual al bien- 
estar colectivo. En todas estas nuevas iniciativas podemos observar un modelo diferente de actuación que los estudios sobre «gobernanza» están intentando integrar dentro de un esquema de análisis coherente. Por último el marco liberal dentro del cual se generan los debates actuales debería ampliarse para permitir que otras corrientes de pensamiento y tradiciones intelectuales puedan verter luz sobre las problemáticas contemporáneas así como sobre las avenidas más prometedoras para el progreso social.

\section{Bibliografía}

Albrow, M. (1996). The global age: State and society beyond modernity. Polity Press, Cambridge.

Alexander, J. (1889). Las teorías sociológicas desde la Segunda Guerra Mundial. Gedisa, Barcelona.

Amato, A. (2006). «La ciudadanía y la sociedad civil ante la Alianza de Civilizaciones», en Barreñada, I. Alianza de Civilizaciones: Seguridad internacional y democracia cosmopolita. Edit. Complutense, Madrid, pp. 222-232.

Anabitarte, A. (2013). «Hacia una sistematización del pacifismo político», en Revista Española de Ciencia Política, $\mathrm{n}^{\circ}$ 31, marzo, pp. 175-189.

Arat F., Z. (1991). Democracy and human rights in developing countries. Lynn Rienner Publishers, Colorado.

Arbab, F. (2000). «Promoting a discourse on science, religion and development», en The Lab, the temple and the market, International Development Research Center, Canada.

Arbab, F. (2006). Evolución de los conceptos de desarrollo. Fundaec, Centro Universitario de Bienestar Rural, Puerto de Tejada.

Arbab, F y Arbab, H. (2006). La elaboración de un marco conceptual para la acción social. Fundaec, Centro Universitario de Binestar Rural, Cali.

Arendt, H. (2005). Sobre la violencia. Alianza, Madrid.

Aristóteles (2004). Ética a Nicómaco. Alianza Editorial.

Ávila-Fuenmayor, F. (2007). «El concepto de poder en Michael Foucault», en REI, $\mathrm{n}^{\circ} 53$, septiembre.

Aymerich Ojea, I. (2001). Sociología de los derechos humanos: Un modelo weberiano contrastado con investigaciones empíricas. Tirant Lo Blanch, Valencia.

Barbieri, K. (2003). The liberal illusion: Does trade promote peace? University of Michigan Press, Michigan.

Bauman, Z. (2003). Modernidad líquida. Fondo de Cultura Económico de Argentina, Buenos Aires.

Beck, U. (2000). La sociedad del riesgo: Hacia una nueva modernidad. Paidós, Barcelona.

Beck, U. (2002). La individualización: El individualismo institucionalizado y sus consecuencias sociales y políticas. Paidós, Barcelona.

Beck, U. (2006). La Europa cosmopolita: Sociedad y política en la segunda modernidad. Paidós, Barcelona.

Bohm, D. (1992). La totalidad y el orden implicado. Kairós, Barcelona.

Boltanski, L y Chiapello, E. (2002). El nuevo espíritu del capitalismo. Akal, Tres Cantos.

Bull, H y Kingsbury (1990). Benedicto and Roberts, Adam, Hugo Grotius and international relations. Oxford University Press, New York.

Camps, V. (2011). El gobierno de las emociones. Herder, Barcelona. 
Chalmers, A. (1999). What is this thing called science? University of Queensland Press, Sidney.

Coser, L. A. et al (1961). Las funciones del conflicto social. Fondo de Cultura económica, México.

Dawkins, R. (2014). El gen egoístas: Las bases biológicas de nuestra conducta. Salvat Ediciones, Barcelona.

Dion, S. (2005). La política de la claridad: Discursos y escritos sobre la unidad canadiense. Alianza Editorial, Madrid.

Domingo, R. (2010). The new global law. Cambridge University Press, New York.

Douglas, M y Wildawsky, A. (1982). Risk and culture. University of California Press. California.

Drahos, P. (2010). The global governance of knowledge: Patent offices and their clients. Cambridge University Press, New York.

Effendi, S. (1089). El desenvolvimiento de la civilización mundial. Ebila, Argentina.

Effendi, S. (1973). La meta de un nuevo orden mundial. Ebila, Buenos Aires.

Eisenstdat, S. N. (1972). Modernización. Movimientos de protesta y cambio social. Amorrortu editores.

Espósito, C. (2005). «Uso de la fuerza y responsabilidad de proteger. El debate sobre la reforma de la ONU», en FRIDE, junio, informe número 3.

Evans, T. (2001). The politics of human rights: A global perspective. Pluto Press, London.

Fergany, N. (2006). «La erradicación de la pobreza a través del buen gobierno: una perspectiva regional árabe», en Barreñada, I. Alianza de Civilizaciones: Seguridad internacional y democracia cosmopolita. Edit. Complutense, Madrid, pp. 64-82.

Fernández, E. M. (1998). El sistema de seguridad colectiva de las Naciones Unidas. Editorial KR, Madrid.

Fishkin, J. (2009). When the people speak: Deliberative democracy and public consultation. Oxford University Press, Oxford.

Foucault, M. (1986). La voluntad de saber. Siglo XXI, Bogotá.

Foucault, M. (2002). Vigilar y castigar. Siglo XXI Editores, Buenos Aires.

Fukuyama, F. (1992). El fin de la historia. Planeta, Barcelona.

García Ruiz, P. (1993). Poder y sociedad: La sociología política en Talcott Parsons. Ediciones Universidad de Navarra, Pamplona.

García, S. (2003). «Análisis de las resoluciones del Consejo de Seguridad ante los mayores casos de violencia política del siglo XX», en Dilemata. International journal of applied ethics, september, $\mathrm{n}^{\mathrm{o}}$ 13, pp. 93-119.

García, S. (2012). «La ciencia y la religión, dos sistemas de conocimiento complementarios: un estudio de caso acerca del discurso sobre ciencia, religión y desarrollo», en Campos, $\mathrm{J}$ y Alcubilla, M. M. (edit.) Ciencia, umanismo y creencia en una sociedad plural. Universidad de Oviedo, Oviedo.

García, S. (2013). «La ciencia y la religión, dos sistemas de conocimiento complementarios: Una reflexión acerca del discurso sobre ciencia, religión y desarrollo», en Religio in Labiryntho, mayo, pp. 383-296.

García, S. (2014). «Un análisis de las resoluciones del Consejo de Seguridad de la ONU ante el principio de la responsabilidad de proteger», en Compé, Revista Científica de Comunicación, Protocolo y Eventos, $\mathrm{n}^{\mathrm{o}} 3$, septiembre, pp. 119-148.

Giddens, A. (2002). Consecuencias de la modernidad. Alianza Editorial, Madrid.

Ginés, S., Lamo de Espinosa, E y Torres, C. (2006). Diccionario de sociología. Alianza Editorial, Madrid.

Girola, L. (2005). Anomia e individualismo: Del diagnóstico de la modernidad de Durkheim al pensamiento contemporáneo. Anthropos Editorial, Madrid. 
González García, J. Mª (2007). «Máquina burocrática y pacto con el diablo: Dos metáforas del poder en la sociología de Max Weber», en Menéndez Alzamora, M (ed.). Sobre el poder. Tecnos, Madrid, pp. 109-140.

Gramsci, A. (1981). Cuadernos de la cárcel. Era, México.

Grüner, E. (2002). El fin de las pequeñas historias: De los estudios culturales al retorno (imposible) de lo trágico. Paidós, Buenos Aires.

Gurrutxaba Abad, A. (1993). «El sentido moderno de la comunidad», en REIS, nº 64, pp. 201219.

Habermas, J. (1998). Más allá del Estado nacional. Trotta, Madrid.

Hall, P. A., Jacoby, W., Levy, J y Meunier, S. (2014). The politics of representation in the global age. Cambridge University Press, Cambridge.

Harper, S. (2000). The lab, the temple and the market: Reflections at the intersection of science, religion and development. IDRC, Ottawa.

Harvey, D. (1998). La condición de la posmodernidad. Amorrortu, Buenos Aires.

Harvey, D. (2012). El enigma del capital y la crisis del capitalismo. Akal, Madrid.

Heler, M. (2007). Jürgen Habermas y el proyecto moderno: Cuestiones de la perspectiva universalista. Editorial Biblos.

Hermanos, J. (1998). El fin de la esperanza. Tecnos, Madrid.

Howard, M. (2001). La invención de la paz. Salvat, Barcelona.

Huntington, S. P. (2002). Choque de civilizaciones. Tecnos, Madrid.

Innerarity, D. (2001). «¿Qué es eso de la gobernanza?», en Tiempo de Paz, nº 100, primavera, pp. 228-233.

Innerarity, D. (2010). La renovación liberal de la democracia. Fundación Ideas, Madrid.

Innerarity, D. (2011). La democracia del conocimiento: Por una sociedad inteligente. Paidós, Barcelona.

Innerarity, D y Solana, J. (2011). La humanidad amenazada: Gobernar los riesgos globales. Paidós, Barcelona.

Innes, J y Booher, D. (2010). Planning with complexity: An introduction to collaborative rationality for public policy. Routledge, New York.

Ball, S. J. (2001). Foucault y la educación: Disciplina y saber. Morata, Madrid.

Karlberg, M. (2004). Beyond the culture of contest: From adversarialism to mutualism in an age of interdependence. George Ronald, Oxford.

Karlberg, M. (2012). «Reframing public discourses for peace and justice», en Korostelina, K. Forming a culture of peace: Reframing narratives of intergroup relations, equity and Justice. Palgrave Macmillan, New York, pp. 11-42.

Kant, I. (2001). La paz perpetua. Longseller, Buenos Aires.

Korten, D. C. (1998). Cuando las transnacionales gobiernan el mundo: No todo lo que brilla es oro. Cuatro Vientos, Santiago.

Lamo de Espinosa, E. (1990). La sociedad reflexiva. Siglo XXI, Madrid.

Lamo de Espinosa, E. (1996). Sociedades de ciencia, sociedades de cultura. Ensayos sobre la condición moderna. Ediciones Nóbel, Oviedo.

Leff, E. (2004). Racionalidad ambiental: la reapropiación social de la naturaleza. Siglo XXI, Madrid.

López, J. T. (1992). Introducción a la economía política. Civitas, Madrid.

Lukács, G. (1967). «Reification and the consciousness of the proletariat», en History \& Class Consciousness, Merlin Press.

Luhmann, N. (2006). Sociología del riesgo. Universidad Iberoamericana, México.

Macpherson, C. B. (2003). La democracia liberal y su época. Alianza Editorial, Madrid.

Mannheim, K. (1987). Ideología y utopía. Fondo de Cultura Económica, México. 
Max-Neef, M. (1998). Desarrollo a escala humana. Icaria, Barcelona.

Mittelman, J. (2006). El síndrome de la globalización. Siglo XXI, Madrid.

Montobbio, M. (2008). «Nuevos paradigmas para la gobernanza global: Democracia desarro1lo-cultura-paz», en Sistemas, n 207 , pp. 23-42.

Moshman, D y Geil, M. (2008). «Collaborative reasoning: Evidence for collaborative rationality», en Thinking and reasoning, vol. 4, $\mathrm{n}^{\mathrm{o}} 3$, pp. 231-248.

Nardin, T. (1985). La ley y la moral en las relaciones entre Estados. Edamex, México.

Ortega, A. (2007). La fuerza de los pocos. Galaxia Gutenberg, Barcelona.

Pogge, T. (2005). La pobreza en el mundo y los derechos humanos. Ediciones Paidós, Barcelona.

Rakodi, C. (2011). «Revisiting religion: Development studies thirty years on», en World Development, vol 39, $\mathrm{n}^{\mathrm{o}} 1$, pp. 45-54.

Ricoeur, P. (1989). Ideología y utopía. Gedisa, Barcelona.

Rodríguez, A. (2011). «Las revoluciones de colores: Una descripción de las estrategias de acción implementadas por los movimientos sociales exitosos», en Revista Española de Ciencia Política, $\mathrm{n}^{\mathrm{o}}$ 26, julio, pp. 127-148.

Rodríguez Fouz, M y Sánchez de la Yncera, I. (2012). Dialécticas de la postsecularidad. Pluralismo y corrientes de secularización. Anthropos, Barcelona.

Rondinelli, D. (1983). Development projects as policy experiments: An adaptive approach to development administration. Methuen, London and New York.

Sampedro, V. (2000). Opinión pública y democracia deliberativa. Istmo, Tres cantos.

Sen, A. K. (2004). Rationality and freedom. Harvard University Press, Massachusetts.

Sen, A. K. (2007). Identidad y violencia: La ilusión del destino. Katz, Madrid.

Slaughter, A. M. (2004). A new world order. Princeton University Press, New Jersey.

Sunstein, C. (2006). Riesgo y razón: Seguridad, ley y medio ambiente. Katz, Buenos Aires.

Toynbee, A. J. (1970). Estudio de la historia. Alianza, Madrid.

Turcotte, M-F. B., Clegg, S. R y Marin, J. (2008). «Enacting ecological collaborative rationality through multiparty collaboration - A case of innovation in governance», en Innovation and Sustainable Development, vol. 3, $\mathrm{n}^{\mathrm{o}}$ 3, pp. 234-261.

Tyler, T. (2011). Why people cooperate: the role of social motivations. Princeton University Press, New Jersey.

Tzvetan, T. (2003). El nuevo desorden mundial: Reflexiones de un europeo. Península, Barcelona.

Wainwright, H. (2005). «Construyendo la democracia participativa desde los movimientos sociales», en Transnational Institute, junio. En: http://www.tni.org/archives/act/2319

Weber, M. (1993). La ética protestante y el espíritu del capitalismo. Península, Barcelona.

Weber, M. (1984). Los tipos de dominación. Centro Latinoamericano de Economía Humana, CLAEH.

Wilson, W. (1918). La guerra-La Paz: La Sociedad de Naciones. Librería Granada, Barcelona. 